\title{
The relationship between corneal hysteresis and the magnitude of intraocular pressure reduction with topical prostaglandin therapy
}

\author{
Daniel R Agarwal, ${ }^{1}$ Joshua R Ehrlich, ${ }^{1}$ Mitsugu Shimmyo, ${ }^{2}$ Nathan M Radcliffe ${ }^{1}$
}

${ }^{1}$ Department of Ophthalmology, Weill Cornell Medical College, New York, New York, USA ${ }^{2}$ Department of Ophthalmology, New York Medical College, Valhalla, New York, USA

\section{Correspondence to}

Nathan M Radcliffe, 1305 York Avenue, New York, NY 10021, USA;

nmr9003@med.cornell.edu

Accepted 2 March 2011 Published Online First 24 March 2011

\section{ABSTRACT}

Aims To evaluate corneal hysteresis $(\mathrm{CH})$ and intraocular pressure (IOP) before and after IOP lowering with prostaglandin analogue (PGA) therapy in medicationnaïve eyes.

Methods In this retrospective study, we included records from 57 consecutive patients with open angle glaucoma who were initiated on PGA. Patients underwent ocular response analyser measurement with IOP assessment at baseline (untreated) and at follow-up (treated).

Results Median follow-up time between IOP measurements was 1.4 (range $0.4-13.5$ ) months. IOP was reduced by $3.2 \mathrm{~mm} \mathrm{Hg}(18.8 \%)$ from 17.0 to $13.8 \mathrm{~mm} \mathrm{Hg}(\mathrm{p}<0.001)$. CH increased by $0.5 \mathrm{~mm} \mathrm{Hg}$ (5.2\%) from 9.7 to $10.2 \mathrm{~mm} \mathrm{Hg}(p=0.02)$. Baseline $\mathrm{CH}$ (but not baseline central corneal thickness) was a significant predictor of the magnitude of IOP reduction, with patients in the lowest quartile of $\mathrm{CH}$ (mean $7.0 \mathrm{~mm} \mathrm{Hg}$ ) experiencing a $29.0 \%$ reduction in IOP while those in the highest $\mathrm{CH}$ quartile (mean $11.9 \mathrm{~mm} \mathrm{Hg}$ ) experienced a $7.6 \%$ reduction in IOP $(p=0.006)$. A multivariate analysis controlling for baseline IOP demonstrated that baseline $\mathrm{CH}$ independently predicted the magnitude of IOP reduction with PGA therapy in both per cent $(B=3.5, p=0.01)$ and absolute $(B=0.6$, $\mathrm{p}=0.02$ ) terms.

Conclusion Although $\mathrm{CH}$ is influenced by IOP, baseline $\mathrm{CH}$ is independently associated with the magnitude of IOP reduction with $\mathrm{PGA}$ therapy.

\section{INTRODUCTION}

Glaucoma is a potentially blinding optic neuropathy that is estimated to affect 60.5 million people worldwide by $2010 .^{1}$ Identified risk factors for glaucoma include older age, lower central corneal thickness (CCT), elevated intraocular pressure (IOP), African-American race and Hispanic ethnicity. $^{2-4}$ Since IOP is the only currently modifiable risk factor for glaucoma, its accurate assessment and successful reduction is crucial to treating the disease.

The current clinical gold standard for measuring IOP is Goldmann applanation tonometry (GAT). Although it has been shown that GAT values are elevated in eyes with thicker corneas, adjustment of IOP for CCT has not been widely adopted due to the variable magnitude of influence of CCT on GAT and the risk of overcorrection. ${ }^{6}$ In addition, there is evidence from large clinical trials to suggest that low CCT may be a risk factor for glaucoma independent of its direct impact on GAT measurement ${ }^{23}$; this may result from either anatomical structure and/or genetic tissue susceptibility. ${ }^{2} 7$
One possible explanation for the failure of CCT to account accurately for GAT measurement inaccuracies is that resistance to corneal deformation during applanation is more related to corneal viscoelasticity or strength, rather than corneal thickness. ${ }^{8}$ Recently, the Ocular Response Analyzer (ORA; Reichert, Corp., New York, USA) has been used to attempt to adjust for viscoelastic corneal properties when measuring IOP. This non-contact tonometer measures several ocular properties including corneal hysteresis $(\mathrm{CH})$ and Goldmanncorrelated IOP (IOPg). CH is defined as the difference between the air-jet pressure at inward and outward applanation and is considered to be a measure of corneal viscous dampening, and thus the cornea's resistance to deformation. ${ }^{9-11} \mathrm{CH}$ has been shown be associated with progressive field worsening in primary open-angle glaucoma (POAG), and lower $\mathrm{CH}$ was shown to be associated with worse visual field damage in patents with asymmetric POAG. ${ }^{9} 12$ Several recent investigations have demonstrated that IOPg may be a precise and replicable alternative to GAT. ${ }^{13-15}$ Accordingly, in a study of $\mathrm{CH}$ and IOP, IOPg may be the preferable measurement for IOP since both $\mathrm{CH}$ and IOPg are obtained objectively and simultaneously from the same machine.

In the Ocular Hypertension Treatment Study, Brandt and colleagues reported that patients with thicker corneas had smaller IOP reduction from topical IOP-lowering medication. ${ }^{16}$ Since $\mathrm{CH}$ may capture different elements from CCT of the cornea's biomechanical nature, we sought to determine if $\mathrm{CH}$ is also a significant predictor of the expected magnitude of IOP reduction with topical IOP-lowering medication. Furthermore, since increasing $\mathrm{CH}$ was previously reported following surgical IOP lowering, ${ }^{17}$ we sought to determine whether IOP lowering with prostaglandin (PGA) therapy is associated with increasing $\mathrm{CH}$.

\section{METHODS}

The study was a retrospective cross-sectional study. One hundred and thirty-eight consecutive patients aged $\geq 18$ years undergoing sequential $\mathrm{CH}$ measurement at the authors' practices in New York City were considered for inclusion in this retrospective study. All patients had a clinical diagnosis of POAG and had documented open angles by gonioscopy within 2 years of inclusion. Patients $<18$ years of age or with a history of corneal disease or keratorefractive surgery were excluded. Consecutively evaluated eligible patients were included in the study if they were naïve to ocular hypotensive 
therapy and were initiated on PGA monotherapy from an untreated baseline. Eyes with a baseline IOPg $>28(n=10)$ were excluded from the study in order to eliminate leverage points and limit the potential for regression to the mean on the study results.

Included patients were seen at two separate visits where ORA measurement and GAT were performed. In accordance with clinic protocol, ORA measurements were performed until a waveform score of 6.5 or better was obtained or until five measurements were obtained per eye; the measurement with the highest waveform score was retained. Patient records were reviewed for demographic data and medication use. Baseline CCT measurements obtained via ultrasound pachymetry (DGH Technology, Exton, Pennsylvania, USA) were recorded from patient charts. For both the baseline and follow-up visit, IOP measured by GAT and IOPg was recorded.

Statistical analyses were conducted using Stata 11/IC (StataCorp LP, College Station, Texas, USA). IOPg was used as the IOP measurement for all statistics presented, unless otherwise specified. Among patients with two eligible eyes, the right eye was chosen to conduct parametric statistical tests, including paired t tests and ANOVA. The Bonferroni correction was used to adjust for multiple pairwise comparisons where appropriate. Generalised estimating equations were used to construct linear models allowing for adjustment of inter-eye correlation among individual patients. All statistical tests were two-sided, with a 0.05 level of significance.

\section{RESULTS}

We included 57 patients with POAG undergoing initial PGA therapy from an untreated baseline. Patient characteristics are presented in table 1 . The mean and median intervals from untreated baseline to PGA-treated follow-up examination were $2.2 \pm 2.5$ months and 1.4 months, respectively (range 0.4-13.5 months). Over this duration, mean $\mathrm{CH}$ increased significantly (9.7-10.2 mm Hg; $p=0.02)$, while IOP decreased significantly $(17.0-13.8 \mathrm{~mm} \mathrm{Hg}, \mathrm{p}<0.001)$.

We plotted the relationship between baseline and follow-up IOP (figure 1), as well as between baseline $\mathrm{CH}$ and the decrease in IOP between examinations (figure 2). A linear relationship was observed between baseline $\mathrm{CH}$ and both the absolute $(\mathrm{r}=0.34, \mathrm{p}=0.01)$ and per cent change $(\mathrm{r}=0.31, \mathrm{p}=0.02)$ in IOP over this interval. Only a weak, non-significant relationship was observed between CCT and absolute $(\mathrm{r}=0.12, \mathrm{p}=0.3)$ or per cent $(r=0.17, p=0.2)$ decrease in IOPg.

In order to determine whether the response to initiation of topical therapy was dependent on baseline $\mathrm{CH}$ and/or CCT, we

Table 1 Patient characteristics

\begin{tabular}{|c|c|}
\hline Categories & Value \\
\hline Number of patients (number of eyes) & $57(109)$ \\
\hline Age (years) & $54.6 \pm 15.4$ \\
\hline Female (\%) & 45.0 \\
\hline Time between examinations (months) & $2.2 \pm 2.5$ \\
\hline ССТ & $542.8 \pm 34.7$ \\
\hline Baseline $\mathrm{CH}$ & $9.7 \pm 1.9$ \\
\hline Follow-up $\mathrm{CH}$ & $10.2 \pm 1.9^{*}$ \\
\hline Baseline IOPg & $17.0 \pm 4.1$ \\
\hline Follow-up IOPg & $13.8 \pm 4.2 \dagger$ \\
\hline
\end{tabular}

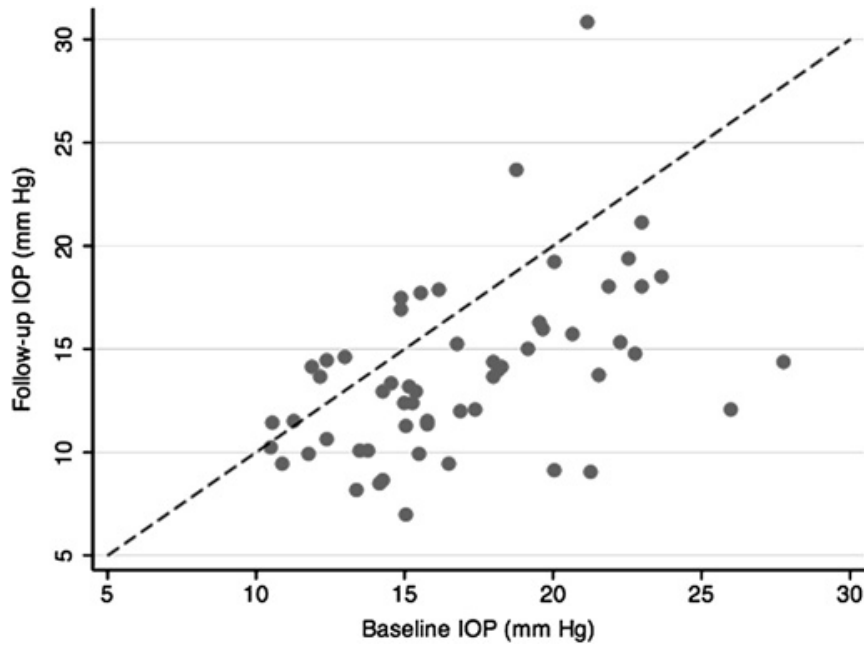

Figure 1 Baseline and follow-up intraocular pressure (IOP). A scatter plot illustrates the relationship between baseline IOP and follow-up IOP for each patient initiated on PGA therapy; the line $y=x$ is superimposed to illustrate the relationship between pre- and post-treatment IOP for each patient.

examined the absolute and percentage decrease in IOP for each quartile of $\mathrm{CH}$ and CCT (table 2). We observed a significantly smaller reduction in IOP among higher $\mathrm{CH}$ quintiles compared with lower $\mathrm{CH}$ quartiles (ANOVA, $\mathrm{p}<0.01$ ). No significant relationship was determined between CCT quartile and response of IOP to topical therapy (ANOVA, $\mathrm{p} \geq 0.4$ ).

Patients with lower baseline $\mathrm{CH}$ also had moderately higher baseline IOP $(r=-0.32, p=0.01)$. Accordingly, we sought to determine whether the greater response to treatment among patients with low $\mathrm{CH}$ occurred due to regression to the mean or whether this relationship existed independently of baseline IOP. Linear models were constructed and after adjusting for baseline IOP, increasing baseline $\mathrm{CH}$ significantly predicted a smaller treatment response as measured by an absolute $(B=0.6,95 \% \mathrm{CI}$ 0.1 to $1.1, p=0.02)$ or per cent $(B=3.5,95 \%$ CI 0.8 to 6.3 , $\mathrm{p}=0.01$ ) change in IOP (table 3 ).

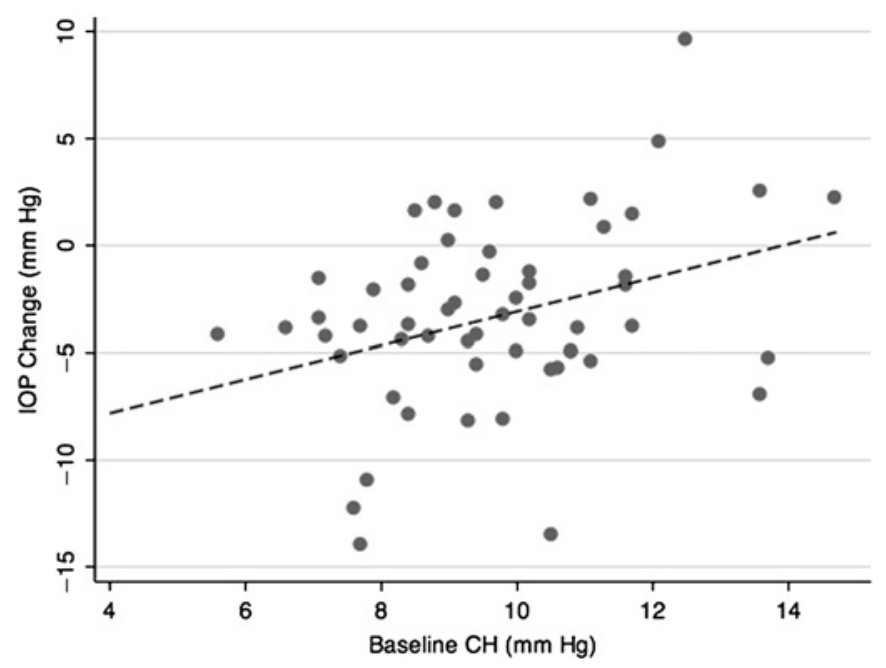

Figure 2 Intraocular pressure (IOP) reduction as a function of corneal hysteresis (CH). A scatter plot illustrates the relationship between baseline corneal hysteresis $(\mathrm{CH})$ and the change in IOP following initiation of prostaglandin analogue (PGA) therapy; the best-fit line is shown $(r=0.5)$. 
Table 2 IOP response to therapy by baseline $\mathrm{CH}$ and CCT

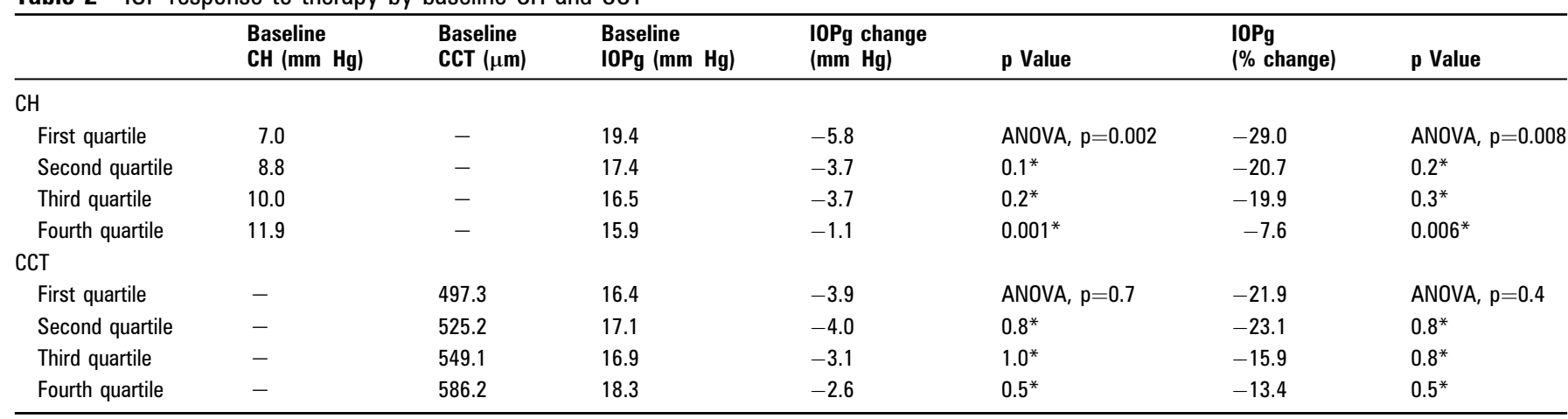

*Pairwise comparisons of change in IOPg compared with first quartile CH or CCT; Bonferroni adjusted significance level of 0.017 .

$\mathrm{CCT}$, central corneal thickness; $\mathrm{CH}$, corneal hysteresis; IOP, intraocular pressure; IOPg, Goldmann-correlated intraocular pressure.

To confirm the validity of our findings using the current clinical standard GAT, we repeated the analyses using this variable and found that GAT decreased significantly with PGA therapy $(p<0.001)$. The magnitude of GAT reduction, as measured by absolute $(p=0.02)$ and per cent change $(p=0.05)$, also decreased with increasing $\mathrm{CH}$. Moreover, we included length of follow-up in the multivariable model containing $\mathrm{CH}$ and IOP and found that it did not significantly alter the relationship between these variables $(p>0.05)$.

\section{DISCUSSION}

In this investigation of patients with POAG experiencing IOP reduction with primary PGA therapy, we confirmed a dependence of $\mathrm{CH}$ on IOP and demonstrated that baseline $\mathrm{CH}$ is associated with the magnitude of IOP reduction. $\mathrm{CH}$ may be a promising metric for predicting glaucoma progression. ${ }^{9}$ The present study adds to past investigations demonstrating that $\mathrm{CH}$ may also be a valuable tool for assessing the expected response of patients to topical PGA glaucoma therapy.

A number of prior studies have examined the association between $\mathrm{CH}$ and glaucomatous visual field or optic nerve findings. ${ }^{9} 1819$ In addition, investigations have indicated that eyes with higher IOP have a lower $\mathrm{CH}$ and that therapeutic manipulation of IOP may induce an inverse response in $\mathrm{CH}^{17}{ }^{20}$ For example, Sun and colleagues found that $\mathrm{CH}$ was significantly lower in eyes with chronic primary angle-closure glaucoma compared with fellow-eyes and normal controls. Moreover, in this prospective study, they found that following trabeculectomy IOP decreased on average from 31.5 to $11.5 \mathrm{~mm} \mathrm{Hg}$ and that $\mathrm{CH}$ increased from 6.8 to $9.2 \mathrm{~mm} \mathrm{Hg}$ over the same time period. $^{17}$

With the apparent dependence of $\mathrm{CH}$ on IOP we expected to observe regression of IOP towards the mean among patients with low $\mathrm{CH}$ in this study. To address the issue of regression to the mean, we adjusted for baseline IOP and determined that $\mathrm{CH}$ remained significantly associated with the magnitude of IOP reduction from topical PGA therapy. Therefore, although $\mathrm{CH}$ is

Table 3 Linear models of relationship between $\mathrm{CH}$ and IOPg response to therapy

\begin{tabular}{lrlc}
\hline & B & 95\% Cl & p Value \\
\hline Outcome: absolute IOPg decrease & & \\
Baseline CH & 0.6 & 0.1 to 1.1 & 0.02 \\
IOPg & -0.5 & -0.8 to -0.3 & $<0.001$ \\
Outcome: per cent IOPg decrease & & \\
Baseline CH & 3.5 & 0.8 to 6.3 & 0.01 \\
IOPg & -1.6 & -2.6 to -0.6 & 0.002 \\
\hline
\end{tabular}

$\mathrm{CH}$, corneal hysteresis; $10 \mathrm{Pg}$, Goldmann-correlated intraocular pressure. generally lower in patients with higher IOP, independently of this a lower $\mathrm{CH}$ may be predictive of a greater IOP reduction from PGA therapy.

Given that a lower $\mathrm{CH}$ is also a risk factor for glaucoma progression and that eyes with worse field damage have lower $\mathrm{CH}$, the finding that patients with lower $\mathrm{CH}$ have a greater response to PGA therapy paints a complex picture in the integration of IOP reduction and baseline risk factors. ${ }^{9} 12$ One possible explanation for this relationship may be that although patients with lower $\mathrm{CH}$ are more responsive to PGA therapy, they require a much greater IOP reduction than those with higher $\mathrm{CH}$ in order to avoid progression. In addition, it is possible that after initiation of therapy clinicians are satisfied with the observed reduction in IOP and do not consider that IOP may be underestimated in patients with lower $\mathrm{CH}$.

In the present study, we were unable to replicate the finding from the Ocular Hypertension Treatment Study that patients with thicker corneas had smaller IOP responses to ocular hypotensive medications including PGAs. ${ }^{16}$ However, in that study Brandt and colleagues found a difference in IOP reduction of only $2.3 \mathrm{~mm} \mathrm{Hg}(8.2 \%)$ between the top and bottom CCT tercile for patients receiving PGA treatment. ${ }^{16}$ Since we did observe a trend towards patients with thicker corneas experiencing a smaller IOP reduction, we believe that the current study may have been underpowered to the relatively small impact of CCT on IOP response to topical therapy.

There are multiple hypotheses that may explain the differential response to PGA therapy as a function of $\mathrm{CH}$ seen in this study. First, as Brandt and colleagues hypothesised regarding patients with thick corneas, ${ }^{16}$ it is possible that patients with high $\mathrm{CH}$ comprise the $15-30 \%$ of glaucoma patients cited as non-responders in drug trials. ${ }^{21} 22$ It is also possible that IOP measurement is affected by $\mathrm{CH}$ in such a manner that changes in IOP are more difficult to measure in eyes with high $\mathrm{CH}$. Since a higher $\mathrm{CH}$ corresponds to greater resistance to corneal deformation, it is not surprising if any form of tonometry dependent on corneal deformation or applantation may be impacted by $\mathrm{CH}^{10}{ }^{11}$ Finally, it is possible that $\mathrm{CH}$ correlates with biostructural features of the cornea, such as collagen composition, that may shape the innate susceptibility of an eye to PGA treatment. The possibility that $\mathrm{CH}$ correlates with such structural features of the eye is supported by research that has shown a decrease of $\mathrm{CH}$ with ageing independent of changes in CCT or $\mathrm{IOP}^{23}$ as well the finding that glaucoma patients with low $\mathrm{CH}$ but not low CCT have greater backward bowing of the lamina cribrosa in response to transient IOP elevation. ${ }^{18}$

There are several limitations to the current study. Principally, the investigation is limited by its retrospective nature. 
Accordingly, we are unable to draw conclusions about causality and the prospective impact of $\mathrm{CH}$ on IOP reduction. In addition, we chose to employ IOPg to measure IOP in this study. Since some prior studies have relied on other measurements of IOP, particularly GAT, this may limit comparability across studies. Nevertheless, we believe that this is a minor point since the outcome of interest in this study was IOP change, not true IOP, so any reproducible method of IOP measurement should provide external validity. Finally, the study is limited by the lack of serial IOP measurements. The authors acknowledge the improved precision of serial IOP measurements; however, there is no reason to believe that noise generated by a lack of serial measurements would bias the study in a particular direction. Acquisition of multiple IOP measurements was beyond the scope of the current study, which is retrospective in nature and exploited extant data from the standard clinical evaluation of patients.

The present study adds to what is already known about the usefulness of $\mathrm{CH}$, demonstrating that this measure of corneal biomechanics may be useful for predicting IOP change in research and/or clinical settings. That $\mathrm{CH}$ is partially dependent upon IOP deserves further consideration in determining whether $\mathrm{CH}$ is truly an innate corneal property. Nevertheless, a prospective study with multiple treatment arms would be useful in better defining this relationship and clarifying its full implications for improving patient care.

Acknowledgements The authors would like to thank the Weill Cornell Medical College Department of Ophthalmology for institutional support.

Funding This work was supported in part by the American Glaucoma Society and the Medical Student Training in Aging Research Program.

Competing interests All authors have completed the Unified Competing Interest form at www.icmje.org/coi disclosure.pdf (available on request from the corresponding author) and declare that: (1) DRA, JRE, MS and NMR have instrument support from Reichert, Inc. for the submitted work; (2) NMR is a consultant to Allergan and Alcon; and (3) the authors have no non-financial interests that may be relevant to the submitted work.

Ethics approval This study was conducted with the approval of the Institutional Review Board at Weill Cornell Medical College and New York Presbyterian Hospital.

Provenance and peer review Not commissioned; externally peer reviewed.

\section{REFERENCES}

1. Quigley HA, Broman AT. The number of people with glaucoma worldwide in 2010 and 2020. Br J Ophthalmol 2006:90:262-7.

2. Leske MC, Heijl A, Hyman L, et al. Predictors of long-term progression in the early manifest glaucoma trial. Ophthalmology 2007:114:1965-72.
3. Gordon MO, Beiser JA, Brandt JD, et al. The Ocular Hypertension Treatment Study: baseline factors that predict the onset of primary open-angle glaucoma. Arch Ophthalmol 2002;120:714-20.

4. Tielsch JM, Sommer A, Katz J, et al. Racial variations in the prevalence of primary open-angle glaucoma: The Baltimore Eye Survey. JAMA 1991;266:369-74.

5. Doughty MJ, Zaman ML. Human corneal thickness and its impact on intraocular pressure measures: a review and meta-analysis approach. Surv Ophthalmol 2000;44:367-408.

6. Gunvant $\mathbf{P}, \mathrm{O}^{\prime}$ Leary DJ, Baskaran $\mathrm{M}$, et al. Evaluation of tonometric correction factors. J Glaucoma 2005;14:337-43.

7. Toh T, Liew SHM, MacKinnon JR, et al. Central corneal thickness is highly heritable: the twin eye studies. Invest Ophthalmol Vis Sci 2005:46:3718-22.

8. Schroeder B, Hager A, Kutschan A, et al. Measurement of viscoelastic corneal parameters (corneal hysteresis) in patients with primary open angle glaucoma. Ophthalmologe 2008;105:916-20.

9. Congdon NG, Broman AT, Bandeen-Roche K, et al. Central corneal thickness and corneal hysteresis associated with glaucoma damage. Am J Ophthalmol 2006;141:868-75.

10. Luce DA. Determining in vivo biomechanical properties of the cornea with an ocular response analyzer. J Cataract Refract Surg 2005;31:156-62.

11. Touboul D, Roberts C, Kérautret J, et al. Correlations between corneal hysteresis, intraocular pressure, and corneal central pachymetry. J Cataract Refract Surg 2008; 34:616-22.

12. Anand A, De Moraes CG, Teng CC, et al. Corneal hysteresis and visual field asymmetry in open angle glaucoma. Invest Ophthalmol Vis Sci 2010;51:6514-18.

13. Vandewalle E, Vandenbroeck S, Stalmans I, et al. Comparison of ICare, dynamic contour tonometer, and ocular response analyzer with Goldmann applanation tonometer in patients with glaucoma. Eur J Ophthalmol 2009:19:783-9.

14. Ehrlich JR, Haseltine S, Shimmyo M, et al. Evaluation of agreement between intraocular pressure measurements using Goldmann applanation tonometry and Goldmann correlated intraocular pressure by Reichert's ocular response analyser. Eye (Lond) 2010;24:1555-60.

15. Kotecha A, White E, Schlottmann PG, et al. Intraocular pressure measurement precision with the goldmann applanation, dynamic contour, and ocular response analyzer tonometers. Ophthalmology 2010;117:730-7.

16. Brandt JD, Beiser JA, Gordon MO, et al. Central corneal thickness and measured IOP response to topical ocular hypotensive medication in the Ocular Hypertension Treatment Study. Am J Ophthalmol 2004;138:717-22.

17. Sun L, Shen M, Wang J, et al. Recovery of corneal hysteresis after reduction of intraocular pressure in chronic primary angle-closure glaucoma. Am J Ophthalmol 2009;147:1061-6.e2.

18. Wells AP, Garway-Heath DF, Poostchi A, et al. Corneal hysteresis but not corneal thickness correlates with optic nerve surface compliance in glaucoma patients. Invest Ophthalmol Vis Sci 2008;49:3262-8.

19. Bochmann F, Ang G, Azuara-Blanco A. Lower corneal hysteresis in glaucoma patients with acquired pit of the optic nerve (APON). Graefes Arch Clin Exp Ophthalmol 2008:246:735-8.

20. Ang GS, Bochmann F, Townend J, et al. Corneal biomechanical properties in primary open angle glaucoma and normal tension glaucoma. J Glaucoma 2008;17:259-62.

21. Noecker RS, Dirks MS, Choplin NT, et al. A six-month randomized clinical trial comparing the intraocular pressure-lowering efficacy of bimatoprost and latanoprost in patients with ocular hypertension or glaucoma. Am J Ophthalmol 2003; 135:55-63.

22. Sellem E, Rouland JF, Baudouin C, et al. Predictors of additional intraocular pressure reduction in patients changed to latanoprost/timolol fixed combination. $B M C$ Ophthalmol 2010:10:10

23. Kamiya K, Shimizu K, Ohmoto F. Effect of aging on corneal biomechanical parameters using the ocular response analyzer. J Refract Surg 2009:25:888-93. 